\title{
Renad Sagdeev: On the Occasion of his 70th Birthday
}

\section{Elena Bagryanskaya $\cdot$ Joachim Bargon}

Published online: 8 November 2011

(C) Springer-Verlag 2011

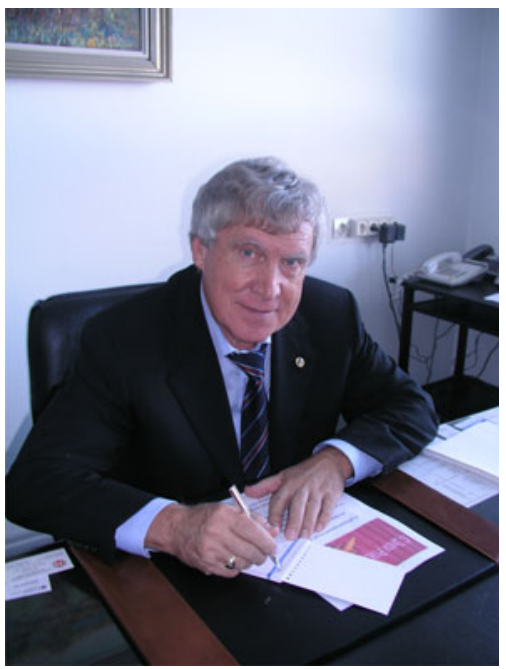

This volume of Applied Magnetic Resonance (AMR) is dedicated to Prof. Renad Sagdeev (International Tomography Center, Siberian Branch of the Russian Academy of Sciences, Novosibirsk, Russian Federation) who celebrates his 70th birthday on December 13, 2011.

\section{E. Bagryanskaya $(\bowtie)$}

International Tomography Center, Siberian Branch of the Russian Academy of Sciences, Novosibirsk, Russian Federation

e-mail: Elena@tomo.nsc.ru

\section{J. Bargon}

Institute of Physical and Theoretical Chemistry, University of Bonn, Bonn, Germany

e-mail: bargon@bonn.de 
With this special issue a good academic tradition is continued to honor scientists who had a major impact in their field. Many of Renad's colleagues and friends, who had been asked to contribute to this special issue, gladly accepted the invitation. Thereby they underline what has been achieved by Renad and his coworkers in the field of spin chemistry, CIDNP application to biological systems, as well as via applications of EPR spectroscopy to materials science and MRI.

Renad Sagdeev was born in Kazan in 1941. He received his diploma degree (in 1966) at Novosibrisk State University, his candidate degree under the supervision of Prof. Yuri Molin (in 1969), and his PhD degree (in 1978) at the Institute of Chemical Kinetics and Combustion of the Russian Academy of Sciences, Novosibirsk, Russia. From 1965 to 1983 Renad worked as a junior research scientist, research scientist, senior research scientist, and head of laboratory of the Institute of Chemical Kinetics and Combustion of the Russian Academy of Sciences (ICKC SBRAS), Novosibirsk, Russia. The fruitful collaboration with Yuri Molin, Kev Salikhov and their colleagues continued over many years and resulted in discovery of magnetic field and magnetic isotope effects, as well as in development of highly sensitive magnetic resonance techniques for detection of short-lived radical pairs. From 1982 till 1993 he was a Deputy Director of ICKC SBRAS. Ever since 1993, Renad Sagdeev is a Director of the International Tomography Center SB RAS at Novosibirsk. In 1992 Renad Sagdeev was appointed as a Corresponding Member of Russian Academy of Sciences, and in 1997 as a Full Member of Russian Academy of Sciences.

Renad Sagdeev has (co)authored more than 400 publications in scientific journals, including 5 books and 10 review articles. His scientific interests include chemical physics, application of NMR and EPR spectroscopy in chemistry, biology and medicine, investigations of the structure and reaction ability of free radicals in solids and solutions, spin chemistry, and molecular magnets. Renad Sagdeev has made outstanding contributions to the fundamental research of multi-spin systems, with particular emphasis on studies of the peculiarities of the electron spin exchange interactions in such systems. The progress achieved by Sagdeev in this field has led to the development of a new type of low-temperature ferromagnetic materials with unprecedented magnetic characteristics based on coordination compounds involving stable nitroxide radicals. Renad Sagdeev in cooperation with his colleagues from ICKC SBRAS and his disciples have contributed decisively to the development of a new field of chemistry-namely the investigation of the influence of electron and nuclear spins on the progress of chemical reactions, which involve short-lived intermediates, such as transient free radicals. The research in this direction has led to the discovery of two new and important phenomena: the influence of static magnetic fields on radical reactions in solutions and the magnetic isotope effect. These phenomena are now recognized as also being of importance for chemical and biochemical processes in living organisms. Renad Sagdeev's efforts were determinant for the foundation of the International Tomography Center of the Siberian Branch of the Russian Academy of Sciences at Novosibirsk, one of the world's recognized leaders in the field of magnetic phenomena in chemistry and medicine. Renad is well known for his optimistic and active lifestyle, and he is a very gifted science manager. He succeeded to organize the new scientific institute in 1993, even 
though this was the hardest time in Russia, and to form a strong scientific team at the time when the immigration of Russian scientists abroad occurred on a mass scale. Renad created very good conditions for successful scientific research of his team, and because of that many have stayed in ITC instead of leaving abroad. As a result, Sagdeev has founded a scientific school and among his disciples are 7 doctors and 15 candidates of science. Most of his followers continue to work in Institutes of RAS up to date.

Renad Sagdeev is a member of a dozen of scientific organizations. He is a Member of the Council for Science, Technology, and Education at the President of Russia (since 2008), Head of the equipment commission of Presidium SB RAS, Member of the Bureau of Joint Scientific Council, on Chemistry SB RAS, Member of the editorial boards of the Russian Journal of Physical Chemistry, Journal of Structural Chemistry, Russian Chemical Reviews, Applied Magnetic Resonance, as well as Distinguished Professor of Moscow State University (1999), Distinguished Professor of Rostov State University (1997), Honorary Professor of Irkutsk State University (2000). He was awarded the Lenin Prize for the investigation of magnetic and spin effects in chemical reactions in 1986, the State Prize of the Russian Federation on the investigation of imidazoline nitroxides in 1994, Order of Honor (2002), Order "Of Service to the Fatherland", 4th Class (2007), etc. Together with Ulrich Steiner and Herhard Closs he initiated Spin Chemistry Meetings which continue to this day. For many years Renad was a member of International Committee on Spin Chemistry, a member of Advisory Board of AMPERE, etc.

We hope that these 34 papers reviewing selected topics will be of great interest to a broad readership of senior and junior scientists. The issue is opened by "A Tribute to Renad Z. Sagdeev on the occasion of his 70th birthday" written by Robert Kaptein, famous scientist in field of spin chemistry due to proposed radical pair theory, "CIDNP Kaptein rules" and 2D NMR applications in studies of proteins structure. The first scientific paper of the issue is the review contribution from wellknown experts in the field of EPR spectroscopy and good friends of Renad for many years Klaus Möbius (Free University of Berlin), Wolfgand Lubitz (Mülheim/Ruhr) and Anton Savitsky (Mülheim/Ruhr). The issue continues with the eight contributions on spin chemistry applications to wide range of chemical and biological problems. The spin chemistry papers are followed by a series of papers related to EPR and NMR applications to biological systems, metal complexes, quantum computing, etc. The last part of issue presents six contributions from instrumentation developments for MRI and MRI application in medicine, including a review of MRI development for human brain studies at $7 \mathrm{~T}$.

One-third of issue is contributed by scientists from International Tomography Center which is founded and directed by Renad. Five papers are presented by scientists of the Institute of Chemical Kinetics and Combustion where Renad had been working for many years. In addition, scientists from Kazan, where Renad was born and grew up, presented papers concerning NMR and EPR applications to study structure and dynamics of organic compounds.

These examples reflect the wide range of scientific problems Prof. Renad Sagdeev has been concerned with, and he still continues to do so, predominately in 
the area of development and applications of magnetic resonance to chemical and biological problems.

Among the authors of papers of this issue there are many well-known scientists who contributed a lot to the development of spin chemistry and its applications, and who had close collaborations with Renad in the past and are his good friends-Yuri Molin, Kev Salikhov, Ulrich Steiner, Henning Paul, Klaus Roth, Gunter Gramp, Malcolm Forbes and many others excellent scientists.

We are grateful to all authors of this special issue who accepted the invitation to contribute. We also thank the referees who provided constructive criticism and thereby contributed to improvement of the submitted manuscripts, and Prof. Kev Salikhov and Dr. Laila Mosina for their support. Last but not the least, we want to join in wishing Renad Sagdeev a happy 70th birthday, good health, and many more years to come. 